\title{
SPATIO-TEMPORAL PATTERN ANALYSIS FOR REGIONAL CLIMATE CHANGE USING MATHEMATICAL MORPHOLOGY
}

\author{
M. Das ${ }^{\mathrm{a}}$, S. K. Ghosh ${ }^{\mathrm{a}, *}$ \\ ${ }^{a}$ School of Information Technology, Indian Institute of Technology Kharagpur - monidipadas@ hotmail.com, skg@iitkgp.ac.in
}

KEY WORDS: Climate Change, Spatio-temporal Pattern, Mathematical Morphology, Directional Granulometry, Multifractal Analysis

\begin{abstract}
:
Of late, significant changes in climate with their grave consequences have posed great challenges on humankind. Thus, the detection and assessment of climatic changes on a regional scale is gaining importance, since it helps to adopt adequate mitigation and adaptation measures. In this paper, we have presented a novel approach for detecting spatio-temporal pattern of regional climate change by exploiting the theory of mathematical morphology. At first, the various climatic zones in the region have been identified by using multifractal cross-correlation analysis (MF-DXA) of different climate variables of interest. Then, the directional granulometry with four different structuring elements has been studied to detect the temporal changes in spatial distribution of the identified climatic zones in the region and further insights have been drawn with respect to morphological uncertainty index and Hurst exponent. The approach has been evaluated with the daily time series data of land surface temperature (LST) and precipitation rate, collected from Microsoft Research - Fetch Climate Explorer, to analyze the spatio-temporal climatic pattern-change in the Eastern and North-Eastern regions of India throughout four quarters of the 20th century.
\end{abstract}

\section{INTRODUCTION}

Modeling climate change has become one of the major research issues as it has serious consequences on human life and ecohydrological processes (Wu et al., 2013). The key challenges here stem from the complex nature of climate data itself. It is because the individual climate variables, governing a climate regime, show very distinctive pattern of change, and the change-pattern also varies from one region to another region throughout the world. Therefore, the regional analysis of climatic pattern change is of utmost significance, particularly for planning adequate mitigation and adaptation measures.

However, the literature mostly concentrates on impact of climate change (Li et al., 2012, Lehodey et al., 2014), than on analysis of climate change pattern. Moreover, the majority of existing works on change pattern analysis (Somot et al., 2008, Gibelin and Déqué, 2003, Mahlstein and Knutti, 2010) are based on global climate models that suffer from the limitations in computing power as well as in proper scientific understanding of physical processes.

In the present work, we have proposed a novel approach for analyzing climate change pattern on regional scale. This is a data driven approach, based on the theory of mathematical morphology, and hence, it intuitively overcomes the inherent limitations of the aforesaid methods. Although the high dimensionality of climate data becomes a major issue in data driven approach, the problem has been tackled by defining a new low-dimensional data set by utilizing multifractal detrended cross correlation analysis (MF-DXA).

\subsection{Contributions}

The proposed approach consists of three main steps: $i$ ) to identify the various climate zones in the study region using a spatiotemporal data mining technique as proposed in (Das and Ghosh, 2015); ii) to detect any regional change in the identified climate zones during each considered time period by utilizing the basic operators in mathematical morphology; iii) to characterize the

${ }^{*}$ Corresponding author, Tel: +91 3222-282332 pattern of climate change, occurred if any, by means of granulometric analysis with four directional structuring elements, oriented in North-South (N-S), East-West (E-W), NorthEast-South West (NE-SW), and NorthWest-SouthEast (NW-SE) directions respectively. The approach has been evaluated empirically with the land surface temperature and precipitation rate data sets ${ }^{1}$ of 20 th century, collected from 240 different locations over all the 12 states in entire Eastern and North-Eastern regions of India. The high resemblance of the simulated climate-change-pattern with that really encountered during the hundred-year period (19012000) in the study area, proves and validates the efficacy of our proposed approach. Thus, the main contributions of this work can be summarized as follows:

- Proposing an approach for analyzing spatio-temporal patterns in regional climate change on the basis of directional granulometry.

- Exploring the theory of mathematical morphology and multifractal analysis in climatological study.

- Experimenting with hundred-year data of land surface temperature and precipitation time series over 240 locations on $0.5^{\circ} \times 0.5^{\circ}$ grid in the study region.

- Drawing insights regarding the pattern of regional climate change using morphological uncertainty index and Hurst exponent.

- Verifying the effectiveness of the proposed approach by an empirical study of analyzing climate change pattern during the 20th century in Eastern and North-Eastern India.

The rest of the paper is organized as follows: In section 2., we have a review of some related works. Section 3. briefly describes the theoretical background behind the work. The proposed approach for climate change pattern detection and analysis has been illustrated in section 4. The results of simulation have been reported in section 5., along with a brief description of the study

\footnotetext{
${ }^{1}$ Data Source: FetchClimate Explorer (Microsoft-Research, 2014)
} 
area and used data sets. Section 6. provides a detailed discussions on the various insights drawn from our model outcomes. Finally, we conclude in section 7.

\section{RELATED WORKS}

The detection and analysis of climate change, especially on a regional scale, is a critically important issue. Several research works for this purpose have been put forward down through the years. This section briefly describes some of the popular works in this regard.

A decision support system has been proposed in (Wilby et al., 2002) for assessing regional climate change using a robust statistical downscaling technique. The use of statistical downscaling helped in rapid development of scenarios with daily surface weather variables under current and future regional climate forcing. The technique proposed in (McCallum et al., 2010), is based on the sensitivity analysis of climate variables using a soil-vegeta tion-atmosphere-transfer model. The approach helped to determine the importance of various climate variables in regional change analysis. Few more relevant studies can be found in (Maurer et al., 2007, Li et al., 2012, Lehodey et al., 2014). However, these works are mainly centered on the analysis of impact of climate change, rather than on the analysis of climate change pattern.

On the other hand, the existing research works on regional climate change pattern analysis are mainly based on the various global circulation models or GCMs (Nobre et al., 1991, Gibelin and Déqué, 2003, Somot et al., 2008, Mahlstein and Knutti, 2010). For example, Nobre et al. in (Nobre et al., 1991) has used a coupled numerical model for a global as well as regional assessment of climate change due to Amazonian deforestation. A sea atmosphere Mediterranean model has been proposed in (Somot et al., 2008), where global atmosphere model has locally been coupled with regional ocean circulation model to study the climate evolution in the Mediterranean region. A number of global coupled atmosphere ocean general circulation models (AOGCMs) have been used in (Mahlstein and Knutti, 2010) to identify the regional climate-change-pattern through a cluster analysis. A variable resolution atmospheric model has been used to simulate the mean sea surface temperature and precipitation field in (Gibelin and Déqué, 2003). However, all these works, being based on global and/or regional climate models, suffers from the limitations of high computing power requirements and incomplete scientific understanding of physical processes in climatology, as mentioned earlier.

Our proposed approach for regional climate change pattern analysis is a data driven approach, and it attempts to overcome the inherent limitations of the existing approaches based on GCMs. Besides, the problem with high dimensionality of climate data, that becomes a major issue in data driven approaches, has been handled here, by defining a new low-dimensional data set by utilizing multifractal detrended cross correlation analysis (MFDXA). Moreover, the novelty of the proposed method lies in analyzing the regional climate change considering the geometric aspects of spatial distribution of various climate zones in the region. To achieve this goal, we have utilized the theory of mathematical morphology followed by granulometric analysis.

\section{BACKGROUND}

This section provides a very brief description of the theoretical background behind our approach, namely, definitions of some basic morphological operators, and multifractal analysis.

\subsection{Mathematical Morphology}

Mathematical Morphology (Serra, 1986) is a powerful tool in image processing, and here, it has been used for analyzing the temporal change in spatial orientation of any climate zone.

3.1.1 Morphological operators: Let $A$ and $B$ be two sets, and $A$ is to be transformed according to $B$. Then, the basic morphological operators can be defined with respect to the structuring element $B$ as follows:

Erosion: Erosion of $A$ by $B$ decreases the size of $A$, and is given by:

$$
A \ominus B=\{a-b: a \in A, b \in B\}=\bigcap_{b \in B} A_{-b}
$$

Dilation: Dilation of $A$ by $B$ increases the size of $A$, which is shown in eq. (2)

$$
A \oplus B=\{a+b: a \in A, b \in B\}=\bigcup_{b \in B} A_{b}
$$

Opening: Opening transformation of the set $A$ by $B$ is given by:

$$
A \circ B=(A \ominus B) \oplus B
$$

Multi-scale Opening: Multi-scale morphological opening can be performed by increasing the size of $B$ as given in eq. (4)

$$
A \circ n B=(A \ominus n B) \oplus n B
$$

where

$$
A \ominus n B=((((A \underbrace{\ominus B) \ominus B) \ominus B) \cdots \ominus B}_{\mathrm{n} \text { times }})
$$

and

$$
A \oplus n B=\left(\left(\left(A_{\mathrm{n} \text { times }}^{\oplus B) \oplus B) \oplus B) \cdots \oplus B}\right)\right.\right.
$$

Closing: Similarly, closing transformation of $A$ by $B$ can be given as follows.

$$
A \bullet B=(A \oplus B) \ominus B
$$

Multi-scale Closing: Multi-scale morphological closing can be performed by increasing the size of $B$ as shown below:

$$
A \bullet n B=(A \oplus n B) \ominus n B
$$

\begin{tabular}{|l|l|l|}
\hline 0 & 1 & 0 \\
\hline 0 & 1 & 0 \\
\hline 0 & 1 & 0 \\
\hline
\end{tabular}

(a)

\begin{tabular}{|l|l|l|}
\hline 0 & 0 & 0 \\
\hline 1 & 1 & 1 \\
\hline 0 & 0 & 0 \\
\hline
\end{tabular}

(b)

\begin{tabular}{|l|l|l|}
\hline 0 & 0 & 1 \\
\hline 0 & 1 & 0 \\
\hline 1 & 0 & 0 \\
\hline
\end{tabular}

(c)

\begin{tabular}{|l|l|l|}
\hline 1 & 0 & 0 \\
\hline 0 & 1 & 0 \\
\hline 0 & 0 & 1 \\
\hline \multicolumn{3}{|c|}{ (d) }
\end{tabular}

(d)
Figure 1. Four directional structuring elements: (a) North-South (N-S), (b) East-West (E-W), (c) NorthEast-SouthWest (NE-SW), (d) NorthWest-SouthEast (NW-SE)

3.1.2 Directional granulometry: In mathematical morphology, the granulometry can be defined as a study of grain-orientation in a binary image (a set). The main principle of granulometry is based on a series of morphological opening operations followed by probabilistic analysis. The granulometric index $H(A / B)$, also called morphological uncertainty index, of a set (binary im- 


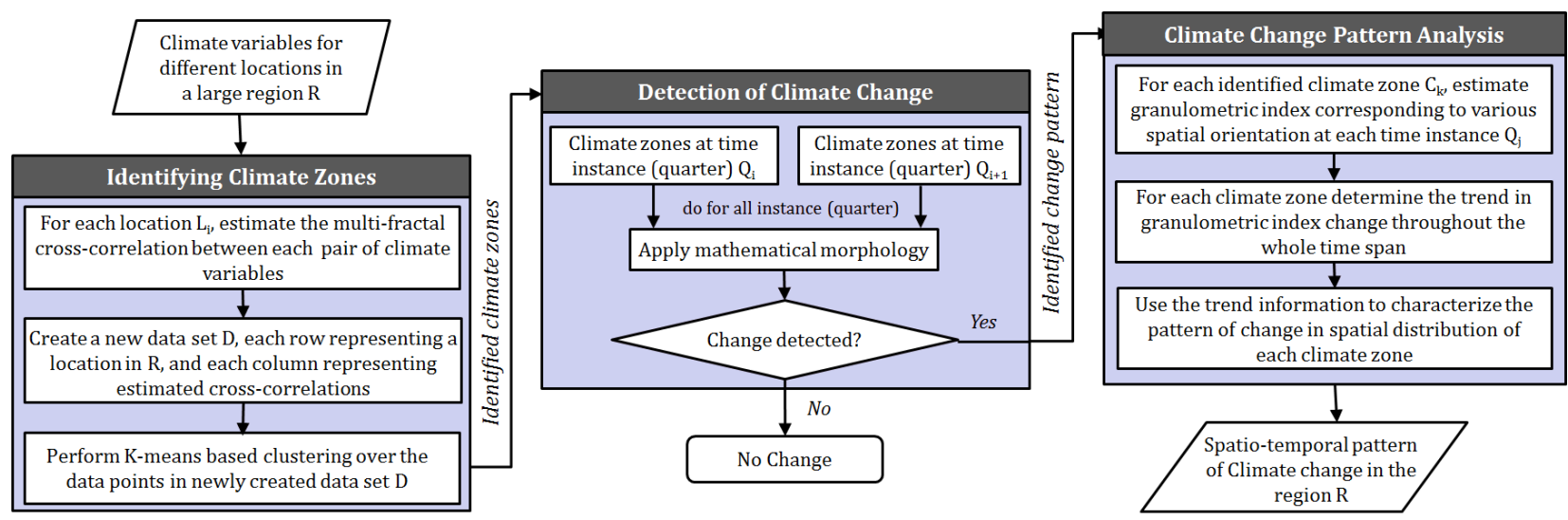

Figure 2. Flow diagram of the proposed approach for regional analysis of spatio-temporal pattern in climate change

age) can be defined as follows:

$$
H(A / B)=-\sum_{n=0}^{N} p_{n} \log p_{n}
$$

where,

$$
p_{n}=\frac{\operatorname{Card}((A \circ n B) \backslash(A \circ(n+1) B))}{\operatorname{Card}(A)}, n=1, \cdots, N
$$

where, $\operatorname{Card}($.$) is the cardinality of the set, and N$ is the minimum positive integer $n$, such that $(A \circ n B) \neq \phi$ and $(A \circ(n+$ 1) $B)=\phi$.

In case of directional granulometry (Vardhan et al., 2013), the structuring element $B$ is a directional structuring element (example shown in Figure 1).

\subsection{Fractals and Multifractals}

Fractals are the never-ending, self-similar patterns that repeat themselves at different scales (Falconer, 2004). These can either be mathematical or natural objects, whose complexity are quantified in terms of a special measure called fractal dimension. Some of the popular definitions of fractal dimensions are: Hausdorff dimension, Box-counting dimension, and Information dimension. In general, fractal dimensions are determined from the regression lines over log vs. log plots of size (or, change in detail) vs. scale. For example, the box-counting dimension $\left(D_{B}\right)$, as defined below, is estimated as the exponent of a power law.

$$
D_{B}=\lim _{\epsilon \rightarrow 0} \frac{\log N(\epsilon)}{\log (1 / \epsilon)}
$$

where, $N(\epsilon)$ is the number of boxes of side length $\epsilon$ required to cover the fractal object.

There are some objects which cannot be described in terms of a single scaling exponent (fractal dimension), and a multitude of fractal dimensions are required to characterize them properly. These are called multifractals. Unlike fractals, the self-similarity in multifractals are scale-dependent. Some common multifractals, found in nature, are the various climatic time series like air temperature, precipitation, humidity etc.

3.2.1 Multifractal Detrended Cross-Correlation Analysis (MFDXA): The MF-DXA method as proposed by (Zhou, 2008) is a multifractal modification of the detrended cross-correlation analysis ( $D X A)$, and quantifies the long-range cross-correlations between two non-stationary time series. Long-range cross-correlations between two series imply that each series has long memory of its own previous values and that of the other series also. If the large and small covariance scales differently, then there will be a significant dependence between detrended cross-covariance $F(q, l)$ and scale length $l$, for different positive and negative values of $q$, which characterizes the multifractality of the series in terms of generalized Hurst exponents $h(q)$ (also called multifractal scaling exponents). The detailed calculations involved in various steps of MF-DXA method can be found in (Zhou, 2008).

The present work uses MF-DXA to detect the climate zones in a large region based on similarity in cross-correlation pattern among different locations in the region.

\section{METHODOLOGY}

Figure 2 presents the basic block diagram of the proposed approach for analyzing spatio-temporal pattern in regional climate change. The system takes as input the historical time series data of various climate variables for different locations in the study region, and finally generates insights regarding the pattern of climate change throughout the past years. As depicted in the figure, the entire system comprises three major steps, namely, (A) Identifying climate zones, (B) Detection of climate change, and (C) Change pattern analysis.

\subsection{Identifying Climate Zones}

The objective here is to capture the long-range cross-correlation between each pair of climate variables $u$ and $v$ over different time scale to characterize the climate pattern of different locations of a region $R$, and then to identify the various climate zones in $R$ on the basis of similarity in spatio-temporal pattern among different locations. The whole estimation is performed in two phases: $i$ ) Multifractal cross-correlation analysis, and ii) Data clustering.

4.1.1 Multifractal cross-correlation analysis: In this phase, a new database, attributing each location, is created. Each tuple/row in the database corresponds to a particular location in the region $R$, and each field/column becomes the multifractal scaling exponents, obtained from multifractal cross-correlation analysis using MF-DXA as follows:

$$
h_{u v}(q)=\frac{\log \left[F_{u v}(q, l)\right]}{\log l}+c
$$

where, $c$ is a constant term. Here, $F_{u, v}(q, l)$ is the q-th order detrended covariance between $u$ and $v$ over a time scale length of l. $h_{u v}(q)$ is also termed as generalized Hurst exponent.The new data set, thus formed, helps to deal with the problem of highdimensionality in data-driven approach. 
4.1.2 Data clustering: Once the data set is created, K-means algorithm is applied to generate the clusters of locations each representing a particular climate zone. Here, the clustering analysis is performed based on the fact that any two locations $l_{i}$ and $l_{j}$ will be in the same climatic zone if the correlation pattern among the same set of climate variables in both the location shows a high degree of similarity.

The details of the climate zone identification procedure can be found in (Das and Ghosh, 2015).

\subsection{Detection of Climate Change}

The main purpose of this step is to detect whether any climatic change has occurred in a region during a particular time period. In general, the spatio-temporal change pattern can broadly be classified into geometric change and thematic change (Ping et al., 2008). The geometric change pattern mainly implies growth, shrinkage, and drift of spatial orientation (Figure 3). In our work, we have considered the first two as the pattern of change. As a growth pattern we have considered spatial expansion, and as a shrinkage pattern we have considered spatial contraction. The other kinds of growth and shrinkage can be spatial merge and split respectively.

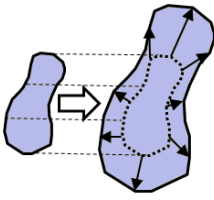

(a)

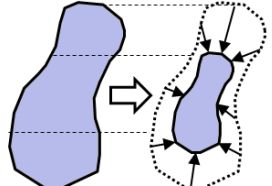

(b)

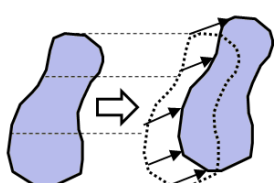

(c)
Figure 3. Change pattern: (a) Growth (expansion), (b) Shrinkage (contraction), (c) Drift

Let $C_{t}=$ Set of all locations under a particular climate zone $C$ at a time instant $t, C_{(t+n)}=$ Set of all locations under a particular climate zone $C$ at a time instant $(t+n)$, Then the conditions for growth, shrinkage, and no-change in spatial distribution pattern of $C$ can be defined as $C_{t} \subset C_{(t+n)}, C_{t} \supset C_{(t+n)}$, and $C_{t}=C_{(t+n)}$ respectively. In our proposed approach, the spatiotemporal pattern of climate change has been detected on the basis of above conditions and utilizing morphological operators as follows:

Small-growth: $\left(\forall_{n \in\left[1, N_{\max }\right]}\left(\operatorname{Card}\left(C_{t} \circ n B\right)<=\operatorname{Card}\left(C_{(t+n)}\right.\right.\right.$ $\circ n B))) \wedge\left(\exists_{n \in\left[1, N_{\max }\right]}\left(\operatorname{Card}\left(C_{t} \circ n B\right)<\operatorname{Card}\left(C_{(t+n)} \circ\right.\right.\right.$ $n B))$ )

Large-growth: $\left(\operatorname{Card}\left(C_{t} \circ\left(N_{\max }+1\right) B\right)=\phi\right) \wedge\left(\operatorname{Card}\left(C_{(t+n)}\right.\right.$ $\left.\left.\circ\left(N_{\max }+1\right) B\right) \neq \phi\right)$

Small-shrinkage: $\left(\forall_{n \in\left[1, N_{\max }\right]}\left(\operatorname{Card}\left(C_{t} \circ n B\right)>=\operatorname{Card}(\right.\right.$ $\left.\left.\left.C_{(t+n)} \circ n B\right)\right)\right) \wedge\left(\exists_{n \in\left[1, N_{\max }\right]}\left(\operatorname{Card}\left(C_{t} \circ n B\right)>\operatorname{Card}\left(C_{(t+n)}\right.\right.\right.$ $\circ \mathrm{nB}))$ )

Large-shrinkage: $\left(\operatorname{Card}\left(C_{t} \circ\left(N_{\max }+1\right) B\right) \neq \phi\right) \wedge(\operatorname{Card}($ $\left.\left.C_{(t+n)} \circ\left(N_{\max }+1\right) B\right)=\phi\right)$

No-change: $\forall_{n \in\left[1, N_{\max }\right]}\left(\operatorname{Card}\left(C_{t} \circ n B\right)=\operatorname{Card}\left(C_{(t+n)} \circ n B\right)\right)$

where, $B$ is a structuring element, $\operatorname{Card}(x)$ is the cardinality of set $x$, and $N_{\max }$ is a positive integer $i$, such that either $\left(C_{t} \circ(i+\right.$ $1) B)$ or $\left(C_{(t+n)} \circ(i+1) B\right)$ becomes $\phi$.

A climate zone is treated to be spreading (growing) if it shows growth pattern during most of the instances in a time duration. Similar decision can be made regarding shrinkage and no-change of a zone. As explained in Figure 2, if no change is detected, the process stops, and no further analysis is performed.

\subsection{Climate Change Pattern Analysis}

Here, the directional granulometry is used to study the spatiotemporal pattern of climate change in the following phases: $i$ ) Granulometric analysis, followed by ii) Pattern categorization.

4.3.1 Granulometric analysis: The objective here is to determine the current spatial orientation of each climate zone at a particular time instant $t$, based on the principles of directional granulometry (Vardhan et al., 2013). Given a climate zone $C_{t}$ at a time instant $t$, the current orientation of $C_{t}$ is estimated as follows:

$$
\operatorname{Orient}\left(C_{t}\right)=\left\{\operatorname{Dir}\left(B^{i}\right): \max \left(H\left(C_{t} / B^{i}\right)\right), \forall i\right\}
$$

where, $\operatorname{Dir}\left(B^{i}\right)$ is the direction of the $i$-th structuring element $B^{i}$, and the $H\left(C_{t} / B^{i}\right)$ is the granulometric index (uncertainty index) corresponding to $B_{i}$, as mentioned in section $3 . . H\left(C_{t} / B^{i}\right)$ is calculated in following manner:

$$
H\left(C_{t} / B^{i}\right)=-\sum_{n=0}^{N_{\max }} p_{n}\left(C_{t} / B^{i}\right) \log p_{n}\left(C_{t} / B^{i}\right)
$$

where,

$$
p_{n}\left(C_{t} / B^{i}\right)=\frac{\operatorname{Card}\left(\left(C_{t} \circ n B^{i}\right) \backslash\left(C_{t} \circ(n+1) B^{i}\right)\right)}{\operatorname{Card}\left(C_{t}\right)}
$$

where, $n$ is the size of structuring element, and $N_{\max }$ is a positive integer such that $\left(C_{t} \circ N_{\max } B\right) \neq \phi$ and $\left(C_{t} \circ\left(N_{\max }+1\right) B\right)=$ $\phi$.

The spatial distribution (orientation) of each climate zone, thus calculated for each time instant, eventually help to achieve insights on the direction of growing (or shrinkage).

4.3.2 Change-pattern categorization: This phase determines the change in spatial orientation of each climate zone from one time instant to another. In other words, it helps to determine the direction of growing (or shrinkage) of the climate zones in a region.

Once the spatial distribution of each climate zone at each time instant is obtained, the characteristics of each pattern is analyzed in terms of trend in temporal change of granulometric indices. Three types of pattern characteristics have been considered: $i$ ) Extinguishing, ii) Evolving, and iii) Stationary. It is evident from eq. 11 that, the more is the value of granulometric index, the higher is the tendency to be oriented in the respective direction. Based on this observation, the pattern of change during a given time period is characterized as follows:

Let, $C_{t}$ be a climate zone (set of locations), orientated towards $B^{i}$ at time instant $t$, and $C_{(t+n)}$ be the same zone with orientation towards $B^{j}$ at time instant $(t+n)$. Also let, the linear trend of change in granulometric index corresponding to $B^{i}$ and $B^{j}$ during the period $[t, t+n]$ is $\operatorname{Trend}^{i}(t, t+n)$ and $\operatorname{Trend}^{j}(t, t+$ $n)$ respectively. Then,

- if $\left(B^{i}=B^{j}\right)$ and $\left(\operatorname{Trend}^{i}(t, t+n)<0\right)$, the orientation of $C_{t}$ in the direction $\operatorname{Dir}\left(B^{i}\right)$ is extinguishing over $[t, t+n]$.

- if $\left(B^{i}=B^{j}\right)$ and $\left(\operatorname{Trend}^{i}(t, t+n)>0\right)$, the orientation of $C_{t}$ in the direction $\operatorname{Dir}\left(B^{i}\right)$ is evolving over $[t, t+n]$.

- if $\left(B^{i}=B^{j}\right)$ and $\left(\operatorname{Trend}^{i}(t, t+n)=0\right)$, the orientation of $C_{t}$ is stationary over $[t, t+n]$. 


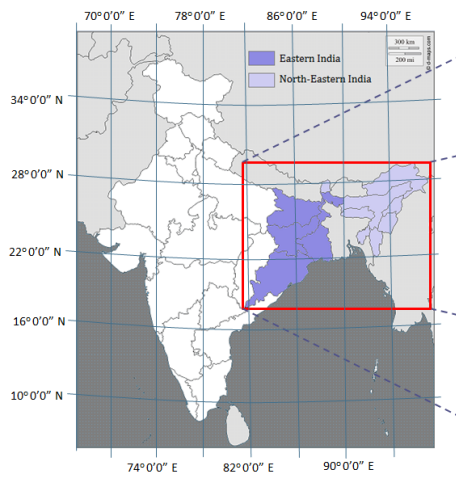

(a)

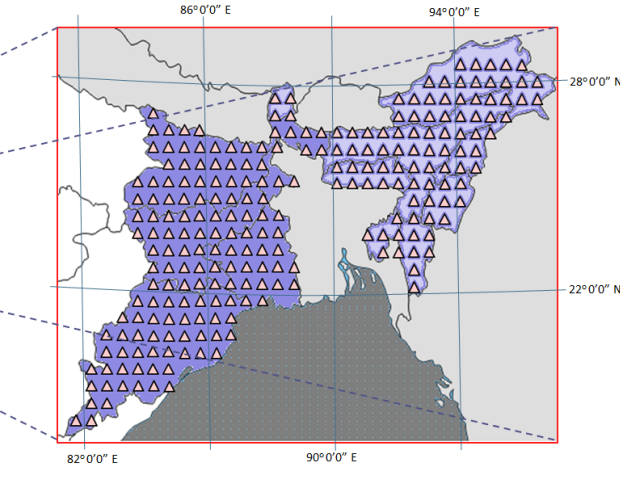

(b)

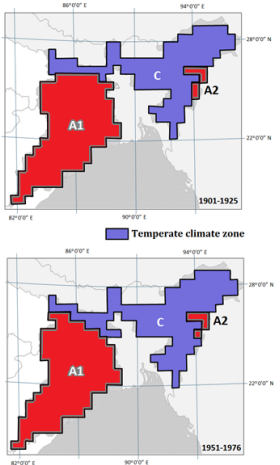

(c)

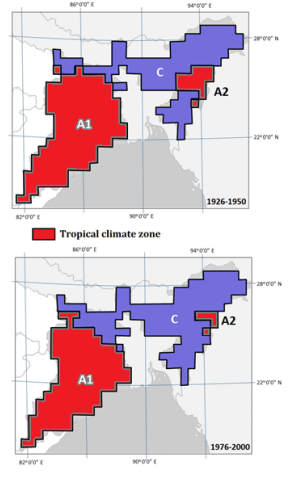

Figure 4. Study area: (a) Eastern and North-Eastern region of India, (b) 240 locations on a $0.5^{\circ} \times 0.5^{\circ}$ grid, (c) Identified climate zones in the study region

- moreover, if $\left(B^{i} \neq B^{j}\right)$, the orientation of $C_{t}$ changes from $\operatorname{Dir}\left(B^{i}\right)$ to $\operatorname{Dir}\left(B^{j}\right)$.

Now, if a climate zone shows a growing pattern along with an extinguishing characteristics, then this indicates its growth not only in its current orientation, but in the opposite direction also. Again, if the zone shows a shrinking pattern along with an evolving characteristics, then this indicates shrinkage in the opposite direction of its current orientation. In the similar fashion, several other inferences can be drawn regarding the spatio-temporal pattern of regional climate-change.

\section{EMPIRICAL EVALUATION}

In this section we have evaluated our proposed approach of climate change-pattern analysis, using an empirical study.

\subsection{Data set and Study area}

The experimentation has been performed using the daily time series data of two major climate variables, namely, land surface temperature and precipitation rate, collected from the Eastern and North-Eastern region of India. As depicted in Figure 4(a), this region of India consists of 12 states. The spatio-temporal data has been collected from 240 different locations on a $0.5^{\circ} \times 0.5^{\circ}$ grid over all these states as pointed in Figure 4(b). The data has been obtained from the FetchClimate Explorer site (MicrosoftResearch, 2014) for a duration of 100 years (1901-2000), in the 20th century.

\subsection{Experimental Setup}

The entire experiment has been carried out using MATLAB 7.12.0 (R2011a) in Windows 2007 (32-bit Operating System, $2.40 \mathrm{GHz}$ CPU, 2.00 GB RAM), and R-tool version 3.1.1 (32 bit). Here, each quarter of the century has been treated as a time instant, and the daily time series data of each climate variable has been averaged over 25-year period to get the representative series for respective quarter.

During the climate zone identification step, the $q$-th order detrended covariance $\left(F_{u v}(q, l)\right)$ vs. scale size $(l)$ has been studied for identifying the pattern of multifractal correlation between the time series of temperature $(u)$ and precipitation rate $(v)$. The study reveals that the multifractal nature of the correlation pattern changes with the climate pattern of a location. The pattern information is then captured in terms of multifractal scaling exponents $h_{u v}(q)$ (generalized Hurst exponent, estimated as the slope of the $\log -\log$ plot for each values of $q$ ), and is utilized to cluster the locations into climate zones. The identified climate zones (Temperate and Tropical) for all the four quarters of the 20th century, and their corresponding patterns of multi-fractal cross-correlation have been depicted in Figure 4(c) and Figure 5 respectively.

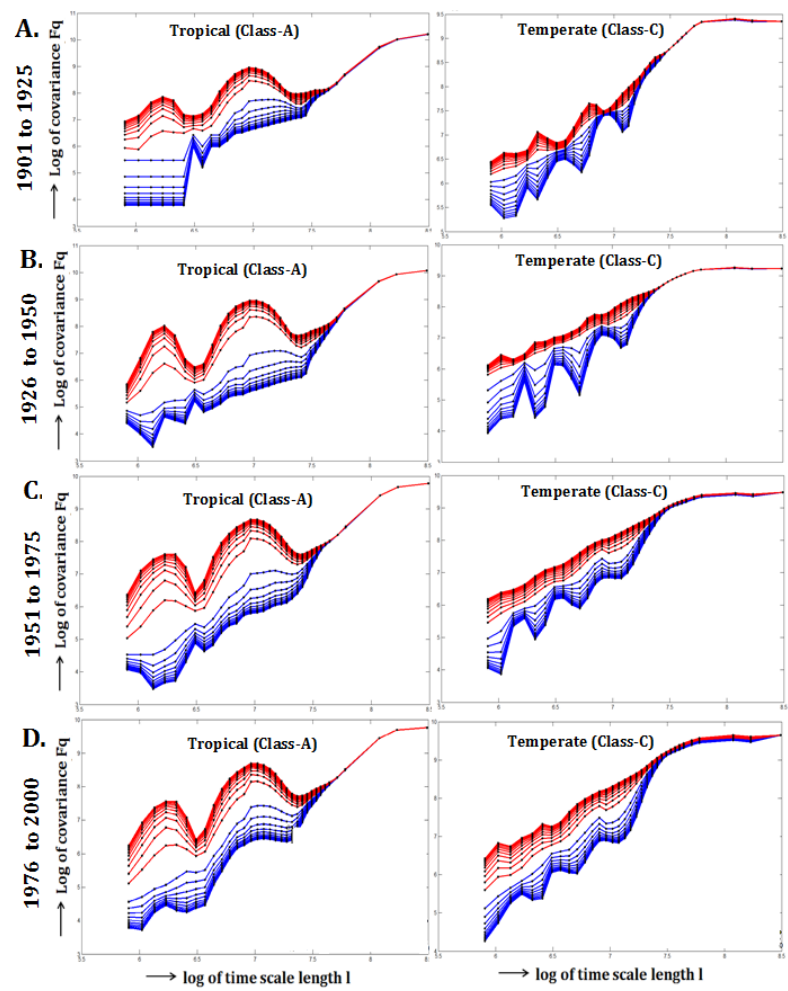

Figure 5. Multifractal cross-correlation pattern of different climate zones

Once the climate zones for each quarter have been identified, the morphological opening is applied between each climate zone and its consecutive instance, to detect whether any growth, shrinkage, or no-change has occurred. In our case study, the tropical zone in the Eastern India has shown shrinkage in most of the quarters during 20th century. Similarly, the temperate zone, and the North-Eastern tropical zone have shown an overall growth and no-change respectively. Then the pattern of climate change throughout the whole century (as depicted in Figures 6, 7,8) is analyzed by performing directional granulometry with four structuring elements, oriented in $N-S, E-W, N E-S W$, and $N W-S E$ direction respectively (Figure 1). 
The temporal evolution of granulometric indices, corresponding to each structuring element, has been plotted in Figure 11 for each part of identified climate zones separately.

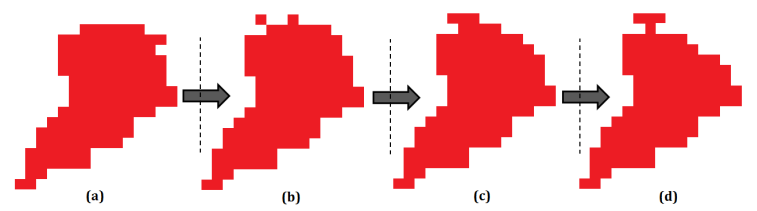

Figure 6. Change in spatial distribution of the Tropical climate zone (A1) in Eastern India: (a)1901-1925, (b)1926-1950, (c)1951-1975, (d)1976-2000

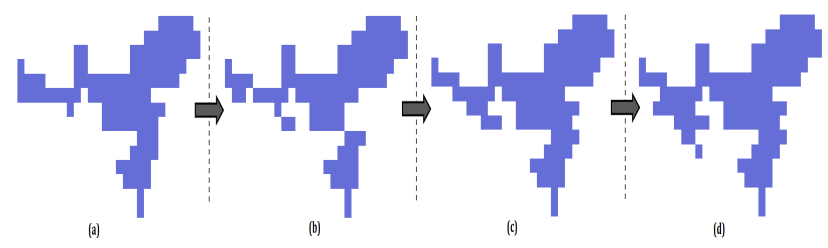

Figure 7. Change in spatial distribution of the Temperate climate zone (C): (a)1901-1925, (b)1926-1950, (c)1951-1975, (d)19762000

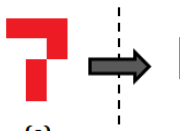

(a)

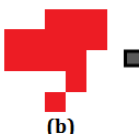

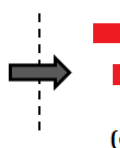

(c)

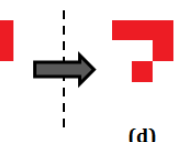

(d)

Figure 8. Change in spatial distribution of the Tropical climate zone (A2) in North-Eastern India: (a)1901-1925, (b)1926-1950, (c) $1951-1975$, (d) $1976-2000$

\begin{tabular}{|c|c|c|c|c|c|c|c|c|c|}
\hline & \multicolumn{2}{|c|}{ 1901-1925 } & \multicolumn{2}{|c|}{ 1926-1950 } & \multicolumn{2}{|c|}{ 1951-1975 } & \multicolumn{3}{|c|}{$1976-2000$} \\
\hline \multirow{3}{*}{$\begin{array}{l}\text { Confusion } \\
\text { Matrix }\end{array}$} & A & $\mathrm{C}$ & A & C & A & C & & A & C \\
\hline & $A^{\prime}[117$ & $15]$ & $A^{\prime}[120$ & $17]$ & $A^{\prime}[117$ & $15]$ & $\mathrm{A}^{\prime}$ & 111 & $11]$ \\
\hline & $C^{\prime}[14$ & 94 & $C^{\prime}[17$ & 86 & $C^{\prime}[3$ & 105 & $C^{\prime}$ & 0 & 118 \\
\hline
\end{tabular}

$\mathrm{A}^{\prime}=$ Tropical climate zone (Actual) $\quad \mathrm{A}:=$ Tropical climate zone (identified) $C^{\prime}=$ Temperate climate zone (Actual) $\quad \mathrm{C}:=$ Temperate climate zone (identified)

Figure 9. Confusion matrices obtained in climate zone identification

\subsection{Performance Evaluation}

The accuracy of climate zone detection has been measured in comparison with the world map of Köppen-Geiger classification (Rubel and Kottek, 2010). Two different performance measures (Overall Accuracy $\left(O_{A}\right)$, and False Alarm Ratio $(F A R)$ ), as shown in Table 1 , have been used for quantitative evaluation. The values of the different parameters are obtained from the confusion matrices (Figure 9) achieved for each quarter of the century.

Performance of climate change pattern detection and analysis has been studied empirically in comparison with the actual climate change encountered during the whole century as shown in the Figure 10.
Table 1. External evaluation of climate zone identification

\begin{tabular}{|c|c|c|c|}
\hline \multirow{2}{*}{ Time Period } & \multirow{2}{*}{$\begin{array}{c}\text { Class } \\
\text { (Climate Zone) }\end{array}$} & \multicolumn{2}{|c|}{ Performance Measures } \\
\hline & & $O_{A}(\%)$ & $F A R$ \\
\hline \multirow{2}{*}{$1901-1925$} & Tropical & 87.92 & 0.11 \\
\hline & Temperate & 87.92 & 0.14 \\
\hline \multirow{2}{*}{$1926-1950$} & Tropical & 85.83 & 0.12 \\
\hline & Temperate & 85.83 & 0.17 \\
\hline \multirow{2}{*}{$1951-1975$} & Tropical & 92.50 & 0.02 \\
\hline & Temperate & 92.50 & 0.12 \\
\hline \multirow{2}{*}{$1976-2000$} & Tropical & 95.42 & 0.00 \\
\hline & Temperate & 95.42 & 0.08 \\
\hline \multicolumn{4}{|c|}{$O_{A}=\frac{T P+F N}{T P+T N+F P+F N} ; F A R=\frac{F P}{T P+F P}$} \\
\hline \multicolumn{4}{|c|}{$\begin{array}{l}T P=\text { True Positive } ; T N=\text { True Negative } \\
F P=\text { False Positive } ; F N=\text { False Negative }\end{array}$} \\
\hline
\end{tabular}

\section{DISCUSSION}

The observations from the empirical study are as follows:

- From the Figures 9 and 4(c), it is evident that two major types of climate zones, namely, Tropical and Temperate, have been identified in the first step, and highly resemble with that in the world map of Köppen-Geiger classification (Rubel and Kottek, 2010).

- The high values of overall accuracy $\left(O_{A}\right)$, and a very low false alarm ratio (FAR), as shown in Table 1 also ensures the efficiency of our approach in climate zone identification.

From the graphical plot of granulometric indices (refer Figure 11 ), the following inferences can be drawn regarding the performance of the proposed approach in climate-change-pattern analysis:

- The tropical zone $A 1$ (refer Figure 11(a)) in Eastern India shows a decreasing trend of granulometric index (uncertainty index) corresponding to its current direction (NE$\mathrm{SW})$. Since, in the change detection step the zone has shown a shrinking pattern, it ensures the direction of shrinkage to be in $N E-S W$. Hence, there must exist a current tendency to be oriented in a different direction. The same is evident from the increasing trend of uncertainty index corresponding to E-W direction (Figure 11(a)).

- It may be noted from Figure 11(b) that, the temperate zone $C$ shows a decreasing trend of granulometric index (uncertainty index) corresponding to its currently prevailing direction (E-W). Since, in the change detection step the zone shows a growing pattern, there must exist a current tendency to grow in the opposite direction (N-S) as well. The same is evident from the actual change in climate as shown in Figure 10(b).

- Therefore, it can be concluded from the above two points that, the type of climate in the North-East part of Eastern India is being changed from tropical to temperate. Thus, the locations in this part of India is going to encounter more hot summer, and a long winter. The same is true for middle East part of North-Eastern India also.

- Conversely, the tropical zone $A 2$ (refer Figure 11(c)) in NorthEastern part of India shows no overall change during the 20th century, and therefore, has a more or less stationary climate pattern. The constant trend $(\approx 0)$ of granulometic index reflects the same. 


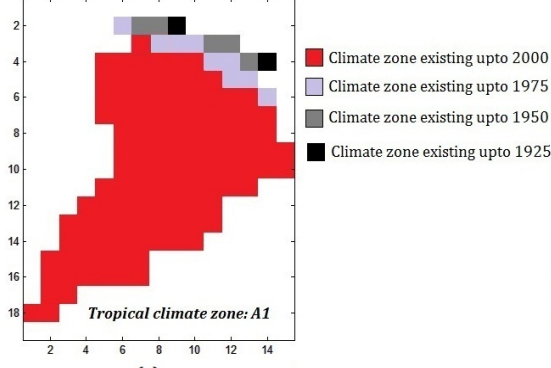

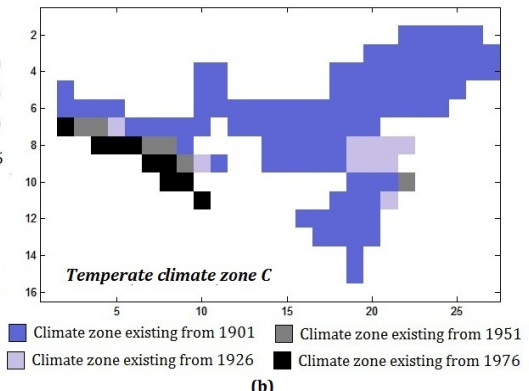

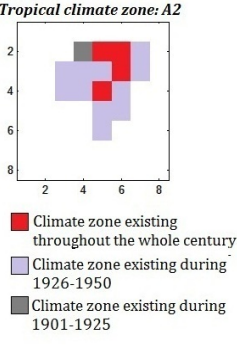

(c)

Figure 10. Spatio-temporal change in climate zones during 20th century: (a) Tropical climate zone (A1) in Eastern India, (b) Temperate zone (C), (c) Tropical climate zone (A2) in North-Eastern India

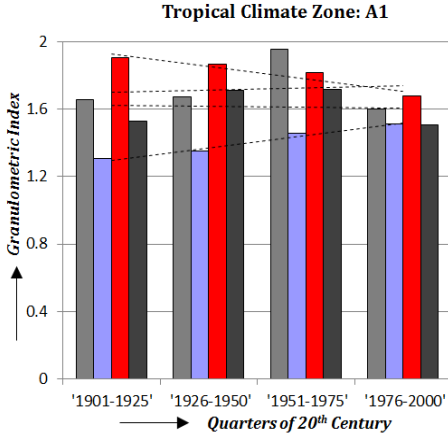

(a)

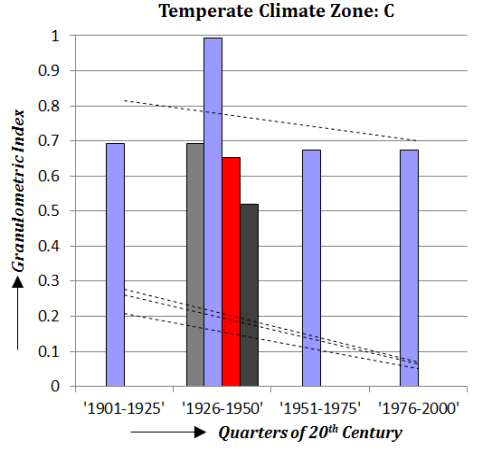

(b)

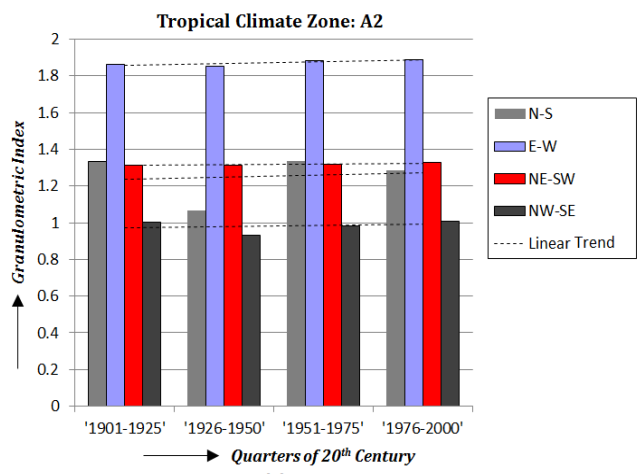

(c)

Figure 11. Change in Granulometric indices (corresponding to various structuring elements) throughout 20th century: (a) Tropical climate zone (A1) in Eastern India, (b) Temperate zone (C), (c) Tropical climate zone (A2) in North-Eastern India

The fifth assessment report of IPCC (IPCC, 2013) supports our outcomes, and demonstrates its efficacy in regional analysis of climate change pattern.

\section{CONCLUSIONS}

This work presents a data driven approach to detect and analyze the regional climate-change pattern on the basis of temporal change in spatial orientation of various climate zones in the region. It has exploited the principles of multifractal analysis and mathematical morphology to identify the climate zones, and to perform the granulometric analysis with four directional structuring elements. A case study has been performed with the temperature and precipitation data of Eastern and North-Eastern region of India, to analyze the spatio-temporal climate-change during 1901-2000. A NE-SW shrinkage of tropical climate zone in the Eastern India, and a growth of temperate climate zone in both $E-W$ and $N-S$ direction have been detected. The high resemblance of the simulated climate-change-pattern with that really encountered during the whole century, proves and validates the effectiveness of applying multifractal and morphological analysis in climate-change-study. This work has considered two basic change patterns: growth and shrinkage, in spatial distribution of climate zones. In future, the work can be extended to deal with the other kinds of patterns like merging, splitting, and drifting or a combination of them.

\section{REFERENCES}

Das, M. and Ghosh, S. K., 2015. Detection of climate zones using multifractal detrended cross-correlation analysis: A spatiotemporal data mining approach. In: Eighth International Conference on Advances in Pattern Recognition (ICAPR), 2015, IEEE, pp. 1-6.
Falconer, K., 2004. Fractal geometry: mathematical foundations and applications. John Wiley \& Sons.

Gibelin, A.-L. and Déqué, M., 2003. Anthropogenic climate change over the mediterranean region simulated by a global variable resolution model. Climate Dynamics 20(4), pp. 327-339.

IPCC, 2013. Climate Change 2013: The Physical Science Basis. http://www.ipcc.ch/report/ar5/wg1/docs/. [Online; Accessed 18-Aug-2014].

Lehodey, P., Senina, I., Nicol, S. and Hampton, J., 2014. Modelling the impact of climate change on south pacific albacore tuna. Deep Sea Research Part II: Topical Studies in Oceanography.

Li, D. H., Yang, L. and Lam, J. C., 2012. Impact of climate change on energy use in the built environment in different climate zones-a review. Energy 42(1), pp. 103-112.

Mahlstein, I. and Knutti, R., 2010. Regional climate change patterns identified by cluster analysis. Climate dynamics 35(4), pp. 587-600.

Maurer, E. P., Brekke, L., Pruitt, T. and Duffy, P. B., 2007. Fineresolution climate projections enhance regional climate change impact studies. Eos, Transactions American Geophysical Union 88(47), pp. 504-504.

McCallum, J., Crosbie, R., Walker, G. and Dawes, W., 2010. Impacts of climate change on groundwater in australia: a sensitivity analysis of recharge. Hydrogeology Journal 18(7), pp. 16251638.

Microsoft-Research, 2014. FetchClimate. http://research. microsoft.com/en-us/um/cambridge/projects/ fetchclimate. [Online; Accessed 28-July-2014]. 
Nobre, C. A., Sellers, P. J. and Shukla, J., 1991. Amazonian deforestation and regional climate change. Journal of Climate 4(10), pp. 957-988.

Ping, Y., Xinming, T. and Huibing, W., 2008. Analysis on geographical changes and research on spatio-temporal patterns. In: XXIst ISPRS Congress Technical Commission II, ISPRS, pp. 1031-1036.

Rubel, F. and Kottek, M., 2010. Observed and projected climate shifts 1901-2100 depicted by world maps of the köppengeiger climate classification. Meteorologische Zeitschrift 19(2), pp. 135-141.

Serra, J., 1986. Introduction to mathematical morphology. Computer vision, graphics, and image processing 35(3), pp. 283-305.

Somot, S., Sevault, F., Déqué, M. and Crépon, M., 2008. 21st century climate change scenario for the mediterranean using a coupled atmosphere-ocean regional climate model. Global and Planetary Change 63(2), pp. 112-126.

Vardhan, S. A., Sagar, B. D., Rajesh, N. and Rajashekara, H., 2013. Automatic detection of orientation of mapped units via directional granulometric analysis. Geoscience and Remote Sensing Letters, IEEE 10(6), pp. 1449-1453.

Wilby, R. L., Dawson, C. W. and Barrow, E. M., 2002. Sdsma decision support tool for the assessment of regional climate change impacts. Environmental Modelling \& Software 17(2), pp. 145157.

Wu, F., Wang, X., Cai, Y. and Li, C., 2013. Spatiotemporal analysis of precipitation trends under climate change in the upper reach of mekong river basin. Quaternary International.

Zhou, W.-X., 2008. Multifractal detrended cross-correlation analysis for two nonstationary signals. Physical Review E 77(6), pp. 066-211. 